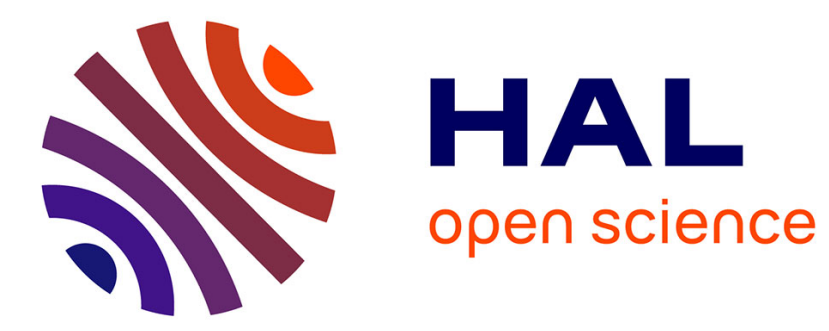

\title{
La torture comme pratique d'Etat dans les pays du Maghreb
}

\author{
Lahouari Addi
}

\section{To cite this version:}

Lahouari Addi. La torture comme pratique d'Etat dans les pays du Maghreb: Régimes politiques et droits humains au Maghreb. Confluences Méditerranée, 2004, 51, pp.141-153. halshs-00398873

\section{HAL Id: halshs-00398873 https://shs.hal.science/halshs-00398873}

Submitted on 25 Jun 2009

HAL is a multi-disciplinary open access archive for the deposit and dissemination of scientific research documents, whether they are published or not. The documents may come from teaching and research institutions in France or abroad, or from public or private research centers.
L'archive ouverte pluridisciplinaire HAL, est destinée au dépôt et à la diffusion de documents scientifiques de niveau recherche, publiés ou non, émanant des établissements d'enseignement et de recherche français ou étrangers, des laboratoires publics ou privés. 
La torture comme pratique d'Etat dans les pays du Maghreb [1]

Lahouari ADDI

Lahouari Addi est professeur des Universités. Il enseigne a l'Institut d'Etudes Politiques de Lyon

In Régimes politiques et droits humains au Maghreb, coordination du dossier paru dans Confluences Méditerrannée, 2004

Sommaire

- $\quad$ Première partie

- Deuxième partie

- Troisième partie

- Quatrième partie

- $\quad$ Cinquième partie

Texte intégral

Même si elle est niée par le discours officiel et interdite par la loi dans les trois pays du Maghreb, la torture, bien présente comme l'attestent de nombreux témoignages, est au cœur de la problématique du politique en ce qu'elle informe sur les rapports réels entre le régime et ses opposants et donc sur le faible degré d'élaboration de l'Etat en tant qu'ensemble de pouvoirs et de contre-pouvoirs institutionnels. La torture n'est pas une pratique policière marginale, ne touchant qu'exceptionnellement une minorité d'opposants. Elle est révélatrice de la nature autoritaire du régime et il suffit qu'un seul administré soit torturé pour que n'importe qui soit susceptible de l'être. Mais pourquoi les Etats du Maghreb n'arrivent-ils pas à interdire dans les faits cette pratique qui discrédite les services de police et qui décrédibilise la justice ? C'est à cette question que tente de répondre cet article.

\section{Première partie}

Le degré de conflictualité et l'intensité de la répression sont différents en Algérie, au Maroc et en Tunisie comme le montrent les articles de ce dossier. Mais les trois pays présentent une caractéristique commune : un déficit important en matière de droits de l'Homme, et particulièrement la pratique de la torture comme moyen de gouvernement. A l'heure de la mondialisation, les habitants des villages les plus éloignés du Maghreb savent qu'il y a des Etats démocratiques où les droits de l'Homme sont respectés, et certains groupes sociaux prennent conscience d'un déficit énorme en la matière. Il est vrai que le problème est d'ordre historique et que le déficit en question renvoie principalement à deux raisons. Ni la communauté ni l'Etat ne reconnaissent à l'individu des droits subjectifs au double niveau religieux et politique. L'appartenance à la collectivité se fait à travers une vision (religieuse) du monde qui fait que l'individu ne peut s'en différencier, ce qui serait perçu comme un détachement et donc une trahison. Cette idéologie holiste conforte la logique autoritaire du pouvoir qui prétend incarner la communauté dans son ensemble et non pas représenter ses membres dans leurs diversités. La conséquence est que l'individu n'est pas un sujet de droit ; la communauté a des droits sur lui et il a des devoirs envers elle, et donc il ne possède pas les droits subjectifs sur lesquels se construit l'Etat de droit. Aussi, quand on analyse le 
fonctionnement des systèmes socio-politiques maghrébins, il n'y a pas à proprement parler de violations de droits de l'Homme pour les dirigeants ; c'est que la notion elle-même semble étrangère à leur culture politique.

Dans la vision du fonctionnaire de l'Etat, ce n'est pas un citoyen sujet de droit qui est torturé, c'est plutôt un administré sur lequel l'Etat a droit de vie et de mort d'où l'agacement des dirigeants face aux rapports des ONG accusées d'«ingérence dans les affaires intérieures d'un Etat souverain». Il est vrai que cet argument est de moins en moins invoqué parce que l'ordre international tient compte de plus en plus des droits de l'Homme. Les Etats maghrébins ont tous adhéré aux conventions et traités internationaux interdisant les violations de droits de l'Homme. Mais ces instruments juridiques ne sont pas respectés parce que les pratiques d'Etat, c'est-à-dire les relations entre les administrés et les titulaires de l'autorité publique, ne sont pas des rapports institutionnalisés obéissant à la règle juridique. Ils obéissent au rapport de forces, dont la pratique de la torture est une des expressions extrêmes.

C'est parce que l'individu est en train d'émerger qu'il y a les manifestations d'intolérance des courants conservateurs religieux et l'autoritarisme d'un Etat se réduisant au pouvoir exécutif. Mais les sociétés maghrébines n'ont pas basculé dans la régulation juridique qui protège l'individu et sa conscience : pour preuve, la pratique de la torture, institution d'Etat qui exprime que l'individu est un administré appartenant au chef auquel il doit prêter allégeance. La torture signifie que l'Etat a droit de vie et de mort sur des sujets sans droits et dont le devoir est de se conformer à la vision du monde qui légitime l'ordre social et politique dominant [2].

L'un des moyens privilégiés des services de police, c'est la torture, dénoncée par les associations locales de droits de l'Homme dont des témoignages accablants sont rapportés régulièrement. Certes, les pays du Maghreb ne sont pas les seuls pays où la torture est pratiquée à grande échelle. Celle-ci est présente dans tout pays non démocratique, où le pouvoir n'est pas issu des urnes et où la Justice n'est pas autonome. Elle est un instrument de gouvernement dans les pays où le régime combat toute opposition qui tente de le remettre en cause. Pratiquée pour faire taire et pour susciter la peur, elle punit ceux qui auront franchi la ligne rouge tracée par le régime et dissuade ceux qui seraient tentés de la franchir. Fondamentalement, elle est un rapport politique violent entre le pouvoir d'Etat et les administrés. Dans cette perspective, elle est un moyen et un attribut de la police pour défendre un ordre politique et social ou même une vision du monde menacés par des individus accusés d'être asociaux, marginaux, ou encore habités par le démon, ou encore soupçonnés d'être des ennemis de la Nation à la solde de l'étranger, bref des ennemis publics dont l'éradication est une oeuvre de bienfaisance.

Les régimes autoritaires ne se contentent pas d'une opinion majoritaire ; ils exigent l'unanimité, se donnant pour fondement l'optimisme anthropologique qui suppose que les hommes sont bons à l'origine. Pour les régimes maghrébins, les citoyens marocains, tunisiens ou algériens sont de bons patriotes, faisant confiance à leurs dirigeants auxquels ils sont fidèles, etc. Mais il y a une minorité qui aurait subi des influences maléfiques - islamisme venu d'Afghanistan, démocratie venue d'Occident... - et qui risque d'introduire le désordre (fitna). II faut alors éradiquer les personnes contaminées pour préserver le patriotisme originel du Maghrébin et la pureté de la communauté. Il suffirait de faire disparaître ces influences et de tuer les personnes contaminées pour revenir au patriotisme originel La violence physique et la torture sont de ce fait des moyens de purification, des instruments pour réaliser la paix sociale ou souder l'unité nationale comme l'enseigne le dirigeant maghrébin. La torture n'est 
donc pas un dépassement d'un policier, un abus de pouvoir localisé d'un gendarme enivré par ses attributions. Elle est une pièce essentielle au mécanisme institutionnel d'ensemble conforté par une vision hiérarchisée de l'ordre politique immuable.

\section{Deuxième partie}

Pour étudier ce phénomène, il faut mettre en œuvre plusieurs dimensions : la psychologie, la psychanalyse, l'histoire, la sociologie politique, l'anthropologie... La psychanalyse aurait certainement beaucoup de choses à dire sur la personnalité du tortionnaire et sur les conséquences de la torture sur le prisonnier. Quand vous sortez de la salle de tortures - si vous en sortez vivant -, vous n'êtes plus le même ; si vous êtes libéré, votre vie sera complètement différente car vous regarderez autrement la société. Je renvoie aux travaux faits par des psychiatres du centre Primo Levi, notamment Françoise Sironi, auteur du livre Bourreaux et victimes, Psychologie de la torture (Odile Jacob, 1999), Je privilégierais cependant l'approche sociologique pour me focaliser sur la torture comme rapport limite d'un système politique avec ses membres, et pour cela je ferai référence à l'histoire, à l'anthropologie, à la sociologie politique...

Des manuels d'histoire nous apprennent qu'elle a fait partie des méthodes archaïques de gouvernement par le passé. Elle a été en effet une pratique de répression médiévale et était une prérogative du pouvoir royal qui avait droit de vie et de mort sur ses sujets. Par le passé, l'ordre politique était considéré comme naturel et se devait de supplicier tous ceux qui le mettaient en cause, en guise de dissuasion, mais aussi pour rétablir l'équilibre rompu par le «délinquant» condamné au supplice. Ce dernier s'appliquait sur des corps supposés possédés par le démon et se déroulait le plus souvent publiquement pour montrer non pas l'inhumanité de la pratique, mais plutôt pour laisser voir la supériorité du Bien sur le Mal, la force de la morale sur ce qui menace l'ordre et la stabilité. Cest ainsi que des souffrances inouïes ont été imposées à des hommes et à des femmes rejetés dans la catégorie d'infra-humains. La conscience du tortionnaire - ainsi que celle du public approbateur - n'était tranquille que parce qu'il était convaincu d'être l'instrument du Bien châtiant le Mal ayant pris une forme humaine. En Europe, le moyen le plus utilisé était celui du démembrement du corps, écartelé par des chevaux faisant tourner des roues opposées. Voltaire, cet ancêtre d'Amnesty International, a mené campagne contre la roue, et aussi contre le fanatisme religieux dont la roue était le symbole physique.

Le fanatisme religieux donne au croyant la certitude du vrai et de la conformité à Dieu et à la morale. Cela signifie que ceux qui ne partagent pas ce même sentiment sont contre Dieu et contre l'humanité ; et qui peut être contre l'humanité si ce n'est le diable ? Cette dynamique d'ethnocentrisme moral libère les passions les plus haineuses menant aux pires atrocités commises au nom du Bien et culminant dans les actes les plus inhumains. Le personnel de l'Inquisition espagnole, en torturant, en démembrant des êtres humains, en assassinant..., avait l'intime conviction qu'il combattait le diable et qu'il purifiait l'humanité de perversions maléfiques. L'intolérance religieuse porte atteinte à la dignité de la personne et provoque aussi des conflits entre groupes appartenant à la même religion dans la mesure où la surenchère sur la pureté de la foi est en corrélation avec la passion et la ferveur religieuses. En religion, écrit Hobbes, «il y aura toujours un plus pur qui épure». Les sociétés musulmanes baignent aujourd'hui dans cette atmosphère de surenchère dans laquelle les hommes, utilisant la religion, cherchent à se hiérarchiser sur le critère de la foi et sur la proximité avec Dieu. Cette idée religieuse de faire le bonheur d'autrui, qui semble être le summum de l'altruisme, n'est 
pas aussi désintéressée qu'il n'y paraît. Elle n'est pas spécifiquement musulmane ; elle est aussi chrétienne et a été même reprise par des régimes prônant l'athéisme comme idéologie d'Etat [3]. Plaire à Dieu, se rapprocher de lui le plus possible est une attitude qui implique que l'on veut être le meilleur des hommes. L'anthropologie humaine est tellement enracinée dans la compétition, dans la distinction (Bourdieu) qu'il faut se demander si l'action altruiste n'est pas une action égoïste plus rusée. En voulant imposer l'ordre moral enseigné par Dieu, en tuant et en assassinant, l'homme se révèle immoral et barbare, s'éloignant de ce qu'il y a de plus humain en lui. Finalement, l'ennemi de l'humanité, ce n'est ni l'animal ni la nature. L'humanité possède en elle un potentiel d'inhumanité qu'exacerbe la passion ethnocentriste, que ce soit sous forme religieuse ou sécularisée. C'est en référence à cette anthropologie que Pascal écrivit que «l'homme n'est ni ange ni bête mais quand il veut faire l'ange il fait la bête».

\section{Troisième partie}

Malheureusement, il n'y a pas que la religion qui peut évoluer vers l'intolérance et la violence. Il y a aussi des idéologies sécularisées qui sont capables de barbarie comme le montrent les exemples du nazisme et du stalinisme avec leurs fours crématoires et leurs goulags. Le nationalisme aussi, quand il s'éloigne de son idée originelle - la concorde nationale, la fraternité communautaire - peut dériver vers l'exclusion en suspectant les uns et les autres d'être des traîtres potentiels ou indignes d'appartenir à la communauté nationale. Au nom de la Nation, construction imaginaire, notion idéelle, on tue des jeunes de vingt ans, on les mutile, on les émascule, on leur crève les yeux, on les ampute des bras et des jambes, on marque leur corps au chalumeau, à la tronçonneuse, à la chignole... tout cela dans des pays qui ont souffert de la domination coloniale combattue les armes à la main [4].

La violence est inscrite dans la sociabilité et est partout imminente quand une idéologie, qu'elle soit religieuse ou politique, se réifie et s'incarne dans des Idéaux érigés en absolu moral auquel se subordonnent - et au nom duquel se sacrifient - des femmes et des hommes. L'histoire des Occidentaux ou des non-Occidentaux est scandée par le rythme des sacrifices morbides pour plaire aux Dieux vindicatifs, avides de chair et de sang. Tuer, torturer, avilir, réduire à néant des hommes pour qu'ils ne contestent pas le monopole qu'a le régime sur la Nation, telle est la mission des services de police pour lesquels la Nation est devenue une idée aliénante dévoreuse d'hommes et de femmes. La Nation ne réunit pas les hommes, elle ne les sert pas, elle s'en sert, se nourrissant de leur chair, de leur sang, de la meurtrissure de leurs corps pendus dans les centres de tortures face aux ricanements des officiers tortionnaires ayant le sentiment de remplir un devoir et de rendre service au pays.

En 1973, après deux tentatives de coup d'Etat infructueuses, le roi du Maroc a déclaré, dans un discours adressé à son peuple, que la tradition islamique l'autorisait à tuer le tiers de la population du royaume pour sauver les deux autres tiers [5], il faut entendre par là qu'il était prêt à déchaîner la pire des répressions s'il lui était demandé de transférer la souveraineté à un Parlement élu sans trucages qui désignerait un gouvernement conformément à la majorité électorale. Les généraux algériens ont mené dans les années 90 une répression à grande échelle sous le prétexte de la lutte anti- terroriste pour dissuader toute contestation qui les remettrait en cause. Ils ne l'ont pas fait au nom de l'islam ; ils l'ont fait au nom de la Nation, estimant qu'ils sont les seuls autorisés à exercer la souveraineté qui leur confère la prérogative de désigner le président et de contrôler le gouvernement. Ils estiment que s'ils venaient à perdre cette prérogative pour en faire un enjeu électoral, l'Algérie en tant que Nation et Etat 
disparaîtrait. Le régime de Ben Ali lance régulièrement des policiers à l'assaut de journalistes, d'universitaires, de militants de droits de l'Homme..., pour les réduire au silence afin de donner l'image d'un peuple tunisien soudé autour du leader éclairé.

Malgré le renouvellement des générations, les régimes maghrébins refusent l'alternance politique, impensable pour eux parce qu'elle porterait atteinte à la Nation menacée de disparition. Une police politique au-dessus des lois a été instituée pour écarter ce danger, pour éliminer physiquement tous ceux qui le portent. Dans le schéma mental du dirigeant, toute opposition menace la Nation et, à ce titre, elle doit être éliminée physiquement à l'instar des islamistes algériens dont le crime a été d'avoir gagné des élections d'où allait sortir une élite investie par l'électorat et non par l'armée. En raison de la victoire électorale d'un parti d'opposition, l'état d'urgence a été proclamé sur tout le territoire de la République jusqu'à ce que le danger islamiste soit écarté. Des ordres précis ont été donnés à tous les services de sécurité - en particulier à la redoutable Sécurité militaire - pour éliminer à jamais les éléments considérés comme les ennemis irréductibles de la Nation. Des milliers de personnes ont été arrêtées pour être exterminées, mais auparavant il fallait les «exploiter», c'est-à-dire les torturer pour tirer toutes les informations possibles sur leurs camarades en fuite. Il fallait aussi dresser les militaires contre les civils, en leur apprenant que ces derniers sont des traîtres potentiels du fait même qu'ils ne portent pas l'uniforme, et cultiver l'esprit de corps pour souder les rangs des militaires se percevant comme les meilleurs fils de la Nation en danger. A partir de là, la torture n'a pas de limites. C'est le schéma médiéval de gouvernement, sauf que dans ce cas il touche des milliers de personnes alors que par le passé il n'en concernait que quelques dizaines.

Les philosophes allemands du XIXème siècle avaient élaboré deux concepts pour décrire cette réalité anthropologique dans laquelle les hommes créent des institutions dont ils deviennent les esclaves ; la réification et l'aliénation. La Nation devient un Dieu avec son culte totémique, ses symboles et son personnel, un véritable clergé pratiquant l'Inquisition avec les attributs de l'Etat moderne. «Pendant d'innombrables millénaires, écrit Cornélius Castoriadis, les sociétés humaines s'auto-instituent - et s'auto-instituent sans le savoir», créant un abîme entre l'image socialement établie du monde et le monde, produisant une réalité imaginaire., un imaginaire social institué, cristallisé dans des abstractions totémiques comme la Nation, la Monarchie, la Religion... pour lesquelles des êtres humains sont tués ou torturés jusqu'à la mort.

\section{Quatrième partie}

La violence physique dans ce cas est utilisée à outrance pour compenser le déficit de légitimité. «Dis à ton Dieu de venir te sauver, Tu es entre mes mains et je fais de toi ce que je veux, ici, c'est moi ton maître. Je vais te niquer. je vais ramener ta femme et ta fille et je vais les niquer devant toi Tu n'es rien, tu es de la merde, etc.». Voilà ce qu'entend le supplicié, ligoté, pendu par les pieds, de la part de son tortionnaire se défoulant sur un corps ensanglanté et dont il est fier d'être le maître, Il y a des témoignages accablants et insoutenables à lire, rapportés dans la machine de la mort par Salah Eddine Sidhoum et Algeria Watch, décrivant les moyens de torture. Ce que le tortionnaire n'accepte pas, c'est que ce prisonnier si vulnérable ait osé s'opposer à l'ordre politique des chefs. Le tortionnaire se croit grandi quand il humilie quelqu'un qui a osé contester politiquement. Il se sent plus courageux qu'un citoyen courageux qu'il brise, qu'il soumet, annihilant sa volonté, pour éventuellement le retourner et en faire un fidèle allié, ou le tuer s'il résiste. C'est cela la feuille de route du tortionnaire qui 
aime dire à son supérieur : «Mission accomplie, chef. La marchandise ne posera plus de problèmes».

Mais d'où vient la force du tortionnaire exerçant une brutalité bestiale sur sa victime ? Il fait partie de la chaîne de commandement d'une institution appelée la force publique dans laquelle il puise ses ressources. Supposons que les amis, la famille ou même la tribu du prisonnier attaquent le centre de torture pour le libérer. La force publique réunira ipso facto des milliers de policiers, gendarmes et, s'il le faut, des militaires avec des chars et des hélicoptères lanceroquettes, fournis par la France et les Etats-Unis, pour faire échouer cette libération. C'est sur cette force que le tortionnaire compte et non sur son propre courage physique ou sa méchanceté. Il a derrière lui un Etat dont la force militaire est supérieure à n'importe quel groupe, organisé ou non, dans la société, Cest pourquoi la torture n'est pas une pratique privée, elle est une institution, elle est une pratique publique, se déroulant dans des endroits publics - commissariats, casernes, centres de détention.... - et exercée par des fonctionnaires payés sur le budget de l'Etat alimenté théoriquement par les impôts des contribuables. Le tortionnaire est un fonctionnaire qui a de l'avancement, des primes de productivité, des congés payés et autres avantages liés à la fonction publique. Il se protège derrière l'anonymat de la fonction publique de telle manière que c'est l'Etat qui torture ; c'est toute l'institution qui torture et lui n'est que l'extrémité d'un mécanisme dont le flux est étatique s'abattant sur une proie dénuée de droits et de statut juridique. C'est pourquoi le tortionnaire n'est pas forcément un pervers, un déséquilibré mental ou un sadique.

Nacéra Dutour, une mère algérienne de disparu, rapporte la conversation qu'elle a eue avec un inspecteur de police au cours de ses multiples démarches pour retrouver son fils ;

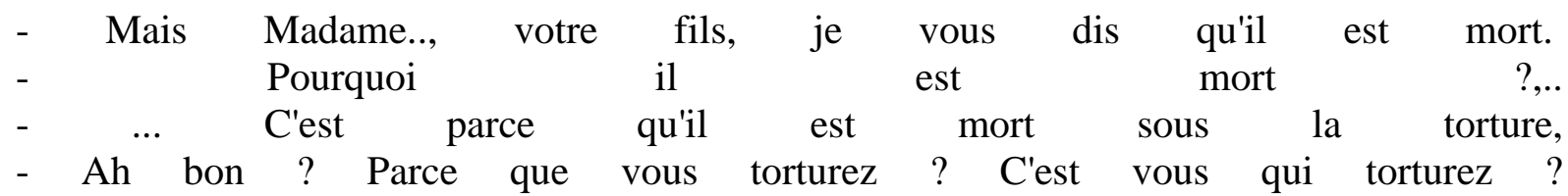

- Non, ce n'est pas moi... Moi mon travail c'est : on m'appelle, je fais des opérations, on me donne une adresse, un appartement, un immeuble ou un endroit, je vais chercher les gens. Je les emmène,..., je les donne en salle de torture... - Vous avez des salles de torture ? - Oh ! Des salles de torture... importées d'Italie ! - Alors... Après ?

- Après, je reviens deux jours après, pour faire le PV, je vois s'ils ont avoué. - Mais s'ils n'ont rien à avouer,.. - Comment ils n 'ont rien à avouer... ? Mais ils ont toujours quelque chose à avouer, toujours. Ils avouent tous, je fais mon PV et je l'envoie au ministère. Au ministère, on me répond : "Vous le donnez à la justice" ou "Il doit mourir" [6]

A travers ce témoignage, l'inspecteur de police apparaît comme un fonctionnaire modèle, fournissant en toute conscience le travail qui est attendu de lui. Souvent, c'est un père de famille,, concevant la torture comme une tâche à accomplir pour défendre l'ordre établi dans le cadre des instructions que ses chefs lui ont données. Et ses chefs, au plus haut sommet de la hiérarchie, ce sont le roi du Maroc, le président algérien et le président tunisien, invités régulièrement en Europe avec les honneurs dus à des chefs d'Etat, comme s'ils étaient totalement étrangers à la torture subie par certains de leurs concitoyens, d'où la pertinence du débat actuel en Algérie et au Maroc sur la Vérité, la Justice et l'Impunité. Reconnaissant à demi-mot ses crimes, l'Etat cherche à garantir l'impunité de ses fonctionnaires grâce au silence des 
familles de disparus et des anciens prisonniers en leur proposant de l'argent public. Il ne s'agit pas de démonter les mécanismes encore intacts des crimes contre l'humanité que sont les disparitions et la torture. Il s'agit seulement de faire taire les victimes et leurs familles par des compensations financières puisées dans le budget de l'Etat en attendant le cycle prochain de la répression massive suivie par une compensation matérielle et ainsi de suite. Ce qui pose problème au régime, ce n'est pas le crime contre l'humanité perpétré par des fonctionnaires contre leurs concitoyens, ce sont plutôt les voix qui le dénoncent et qui informent l'opinion publique internationale. Ces voix qui salissent l'image du pays dans les médias étrangers, il faut les faire taire à tout prix, y compris en les payant. Le président algérien a dit en public à des mères de disparus l'implorant de les aider à retrouver leurs enfants morts ou vivants : «Taisez-vous, les pleureuses. Vous faites honte à votre pays. Vos photos sont dans les médias étrangers à chaque fois que je fais un déplacement à l'extérieur».

\section{Cinquième partie}

Dans le Maghreb colonial, la torture était assez pratiquée contre les indigènes qui contestaient de manière violente la hiérarchie statutaire de la domination française qui avait légalisé l'infériorité des Maghrébins dans leurs propres pays. On aurait pu penser que le Maghreb indépendant bannirait la torture du fait que les Maghrébins en avaient souffert. Que non ! Elle va être utilisée à une échelle industrielle, en particulier en Algérie après janvier 1992, au point que des milliers de personnes sont mortes sous la torture faisant aujourd'hui partie de ces fameux disparus sur lesquels les familles recherchent désespérément la vérité.

Certes, la torture n'est nulle part revendiquée et assumée et aucun dirigeant ne reconnaît officiellement sa pratique. Le discours officiel accuse même les ONG et les militants de droits humains de menées subversives visant à ternir l'image du pays à l'étranger. Les régimes la nient en bloc et les diplomates ne cessent de répéter que les Etats du Maghreb ont signé la convention de l'ONU de 1984 l'interdisant totalement. Le discours officiel la condamne mais les polices continuent de la pratiquer dans les centres de détention en Tunisie, au Maroc et en Algérie. Juridiquement, elle est interdite, mais tout le monde sait qu'elle est pratiquée par tous les corps de sécurité. Les personnes qui aujourd'hui courent le plus de danger, ce sont les militants de droits de l'Homme et les journalistes qui rapportent leurs activités, si bien que l'on peut être torturé pour dénonciation de la torture !

Il est vrai que le fossé, entre d'une part les régimes, monopolisant et privatisant l'idée de Nation, et d'autre part la population aspirant à participer aux affaires de l'Etat, le fossé entre la légalité des forces de police et la légitimité du peuple est si grand que ces régimes tomberaient comme un fruit mûr s'ils n'utilisaient pas la torture pour dissuader et pour contraindre. N'étant pas l'émanation du peuple, ces pouvoirs ont choisi d'être craints pour se maintenir. C'est ainsi que la torture est érigée en moyen de gouvernement pour refuser l'alternance électorale et réprimer toute expression politique légitime, qu'elle soit islamiste ou démocrate. C'est pourquoi il est vain d'espérer la disparition de la torture tant que la démocratie n'a pas vu le jour et que la justice n'est pas indépendante du pouvoir exécutif, c'est-à-dire tant que le pouvoir - aujourd'hui reposant sur la force des armes - n'est pas institutionnalisé et tant que l'autorité ne procède pas de la légitimité populaire. Seule l'institutionnalisation du politique est susceptible d'humaniser les luttes et les conflits entre les intérêts divergents des uns et des autres inhérents à toute société. La contestation politique découle de l'antagonisme irréductible entre gouvernants et gouvernés et ne peut être pacifique que si ceux-ci choisissent ceux-là. Autrement, le régime ne se maintiendra qu'au prix de la terreur imposée par les 
dirigeants qui, pour surmonter leur peur, ont besoin de faire peur à la population, utilisant entre autres la torture à grande échelle. Schématiquement, il y a aujourd'hui deux types de régime : ceux qui, selon $\mathrm{H}$, Arendt, appartiennent au pré-politique et ceux qui appartiennent au politique. Un régime politique, explique la philosophe allemande, est une organisation de la cité construite sur un minimum consensuel excluant la violence physique pour acquérir le pouvoir ou s'y maintenir. Un régime pré-politique est une organisation non institutionnalisée et non consensuelle du pouvoir reposant pour l'essentiel sur la force des armes. Dans le régime politique, la torture est un crime condamné en tant que tel ; dans le régime prépolitique, elle est un instrument de gouvernement et un moyen de coercition parmi d'autres.

La politique est une activité conflictuelle par nature, et elle ne s'humanise que si elle est institutionnalisée dans le cadre de l'Etat de droit où l'électorat est reconnu dans les faits comme le seul détenteur de la légitimité populaire, déléguée périodiquement à des représentants élus, mandatés pour faire des lois et les abroger au nom du peuple souverain. Cette philosophie sur laquelle repose l'Etat de droit suppose cependant une transformation culturelle décisive apportée par la modernité: la société est source de pouvoir, à l'exclusion de toute croyance sacralisée posée au-dessus de la volonté des hommes : la Religion, la Nation la Monarchie, la République, l'Histoire... élevées au rang d'un Dieu implacable et sans merci. C'est pourquoi les islamistes, aujourd'hui victimes de massives violations de droits de l'Homme, sont susceptibles de reproduire les mêmes pratiques tortionnaires s'ils continuent à sacraliser la politique, à présenter leurs adversaires comme des impies ayant trahi la parole de Dieu, ils sont eux aussi susceptibles de torturer leurs anciens tortionnaires et au-delà, tous ceux qui les désapprouveraient. La torture est structurellement constitutive d'un rapport politique qui sacralise les institutions et qui privatise les pouvoirs publics. La modernité fait prendre conscience du danger d'être esclaves d'institutions assoiffées de sang et avides de victimes expiatoires sacrifiées dans le délire collectif. La modernité tente de mettre fin à cet esclavage en posant qu'aucune doctrine, aucune idéologie ne peut être morale si elle ne proclame pas que l'homme est une fin en soi et non un moyen. Cela veut dire qu'aucune doctrine nationaliste n'est morale si elle torture des êtres humains, qu'aucune religion n'est respectable si elle tue des hommes et des femmes accusés d'avoir déplu à Dieu.

Mettre la dignité de l'homme au centre du politique, c'est donner à l'Etat sa dimension humaine en désacralisant les institutions et les constructions imaginaires qui hantent les esprits. Torturer un jeune homme, l'assassiner, le mutiler... au nom de la Nation, de la Monarchie ou de la République, c'est porter atteinte à la communauté et à toute l'humanité, car la Nation est une abstraction si elle n'est pas incarnée par chaque citoyen. C'est quoi une Nation si elle n'est pas déposée dans le corps des êtres humains qui sont supposés la constituer, si les «nationaux» n'étaient pas les parties physiques qui la composent ? C'est quoi la République tunisienne ou algérienne, si les Tunisiens ou les Algériens ne choisissent pas librement leurs représentants ? C'est quoi la monarchie marocaine si les Marocains ne s'y reconnaissent pas?

Notes

[1] Cet article est le texte d'une conférence prononcée en mars 2004- à l'Institut d'Etudes Politiques de Lyon organisée par les étudiants membres d'Amnesty International. 
[2] Les penseurs de la modernité - Hobbes, Grotins, Locke, Rousseau... - ont eu recours au droit naturel pour construire la théorie des droits subjectifs de la personne, insistant sur le fait que l'individu a des droits naturels à sa naissance et qu'il est libre de toute allégeance politique ou sociale qu'il n'accepte pas volontairement Certains diront que l'islam s'opposerait à une telle évolution. Ce serait prêter à la religion un rôle déterminant et structurant les rapports sociaux,

[3] C'est parce qu'il a repris à son compte cette idée religieuse que le marxisme a sombré dans le goulag et autres camps d'épuration.

[4] Le colonel Amirouche de la Wilaya III avait, lors de la "bleuite", fait arracher des aveux à des maquisards sachant lire et écrire le français, soupçonnés de ce fait d'envoyer des renseignements à l'ennemi. La Kabylie a ainsi perdu des centaines de cadres nationalistes instruits, tués par leurs camarades de combat. «Et si certains d'entre eux étaient innocents, mon colonel» avait demandé un subordonné à Amirouche. «Ils iront au Paradis», rétorqua ce dernier. Le monde des exaltés et des aliénés est binaire : soit le Paradis, soit l'Enfer. Le monde réel n'y a pas sa place ; ou bien nous sommes les anges que les gouvernants nous imposent d'être, c'est-à-dire des êtres dociles, obéissants et surtout désintéressés, ou bien nous sommes des diables qu'ils réduisent au silence pour ne pas contester et revendiquer. La torture donne une cohérence à ce monde manichéen en servant de lieu de passage entre l'Enfer réel et le Paradis imaginaire.

[5] Cette tradition est l'œuvre de fouqahas (jurisconsultes) inspirés par Ibn-Taymiya pour qui un Prince injuste est préférable à la fitna, au désordre. Elle n'existe cependant ni dans le Coran, ni dans la Sunna. Il faut remarquer, par ailleurs, que cette même tradition a fait partie de la culture politique européenne médiévale.

[6] Témoignage "de Nacéra Dutour dans Habib Souaïdia, Le procès de "La sale guerre", La Découverte, 2003, p. 341 